$\Rightarrow$ POLYMER CHEMISTRY

\title{
Dial in your dispersity
}

\section{C6 \\ We needed \\ them to be \\ 'bad' enough \\ to give us high \\ dispersity}

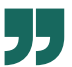

Inspired by the perfect monodispersity of proteins, polymer research has long favoured methodologies that afford products with narrow molecular weight distributions. However, high dispersity polymers are ostensibly more processable and are widely used in industry. We surprisingly do not have good routes to these materials because of the misconception that high dispersity must come at the expense of 'livingness' - the propensity of polymer chains to undergo further reactions. A team led by Athina Anastasaki and Tanja Junkers put paid to this with an unconventional controlled radical polymerization method that exploits multiple chaintransfer agents, as described in Chem, to tune dispersity of a living polymer over a wide range.

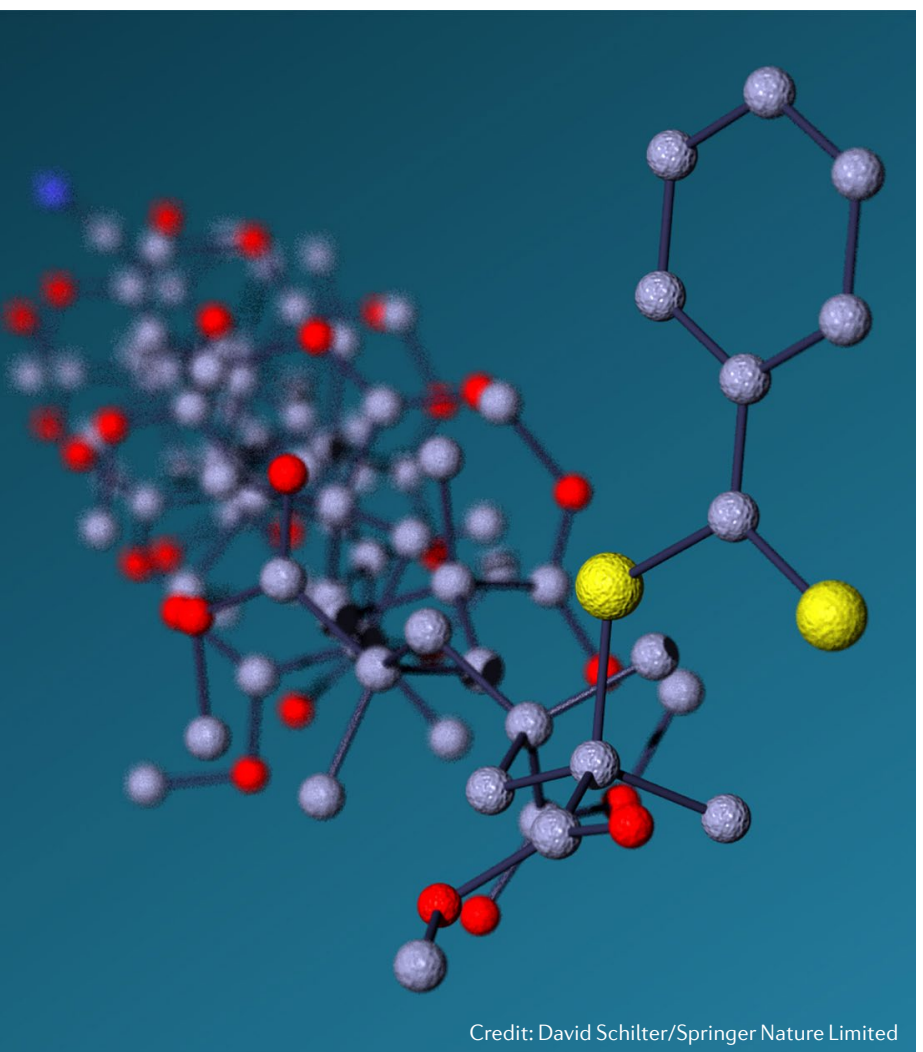

Synthetic linear polymers typically exist as mixtures of species that can vary in their degree of polymerization and the nature of their end groups. The former is described by dispersity $(\nexists)$, the quotient of the weight average to the number average molecular weight and a value that greatly affects physical properties. Historically, high $Ð$ polymers have had limited livingness because not all chains have the requisite active end groups at which to uniformly grow another polymer domain or attach the polymer to a surface. One route to vinyl polymers with high end-group fidelity is reversible additionfragmentation chain transfer (RAFT) polymerization, whereby a growing polymer radical $\mathrm{P}_{n}$ reversibly adds to a chain-transfer agent $\mathrm{ZC}(\mathrm{S}) \mathrm{SR}$ ( $\mathrm{Z}$ is an aryl/heteroatomic group) to give the $\mathrm{C}$-centred radical intermediate $\mathrm{ZC}\left(\mathrm{SP}_{n}\right) \mathrm{SR}$. This product then liberates either the $\mathrm{S}$-bound $\mathrm{P}_{n}$ or $\mathrm{R}$ radical, so one chain continues to grow by reacting with monomer molecules while the other chain takes a break on the agent.

RAFT is geared towards low $Ð$ polymers and if we want to increase this value we could simply initiate chain growth at different times. Yet, realizing this with flow or light is non-trivial, so Anastasaki, Junkers and colleagues considered the chemistry of chain-transfer agents, beginning with alkyl dithiobenzoate $\mathrm{PhC}(\mathrm{S}) \mathrm{S}$ (2-cyano-2-propyl), which enables rapid chain-transfer via a benzylic radical intermediate. This archetypical agent gave poly(methyl methacrylate) with a number average molecular weight of $25,300 \mathrm{Da}$ and a low $Ð$ of 1.13 , so the team sought worse agents to realize higher $Ð$. "We needed them to be 'bad' enough to give us high dispersity but not at the cost of slow polymerization, dead polymer chains or a mismatch between experimental and theoretical molecular weight," recalls Anastasaki. One such 'bad' agent for methacrylates is the alkyl dithiocarbamate [1-(4-chloro3,5-dimethylpyrazolyl)]C(S) $\mathrm{S}$ (2-cyano-2-butyl), which is slower to transfer chains and gives $Đ=1.65$ under the same conditions.

With 'good' and 'bad' agents in hand, how do we arrive at an intermediate dispersity? Anastasaki, Junkers and colleagues show that simply mixing two different agents affords $\boxplus$ between the two extremes, depending on the ratio of agents used. Size-exclusion chromatography reveals a monomodal product distribution, consistent with the growing chains spending time on both agents. Overall, the team's seven agents enabled them to tune the dispersity of polymethacrylates, polyacrylates, polyacrylamides, polystyrene, poly(vinyl ketone) and poly(vinyl acetate).

Although convenient and amenable to a broad substrate scope, the mixed-agent method does have limitations. "The brutal truth is that we can control dispersity very well but not the shape of the molecular weight distribution, which also affects properties," laments Anastasaki. Mass spectrometry shows that they get a mixture of living polymers, $\mathrm{Z}^{1} \mathrm{C}(\mathrm{S}) \mathrm{SP}_{n}$ and $\mathrm{Z}^{2} \mathrm{C}(\mathrm{S}) \mathrm{SP}_{n}$, that feature the two different agents as end groups. This mixture can be converted into diblock copolymers but without perfect control of overall dispersity. However, if we could shape product distributions and have uniform end groups, the method may well enable unprecedented control over polymers and their remarkable properties.

David Schilter

ORIGINAL ARTICLE Whitfield, R. et al. Tailoring polymer dispersity by RAFT polymerization: a versatile approach. Chem https://doi.org/10.1016 j.chempr.2020.04.020 (2020) 